\title{
Diurnal variation in forced vital capacity in patients with fibrotic interstitial lung disease using home spirometry
}

\author{
To the Editor:
}

Forced vital capacity (FVC) is used as the routine physiological measure to assess disease progression in fibrotic interstitial lung diseases (f-ILDs) [1]. New drugs are currently being investigated on top of "standard care" with antifibrotic drugs in idiopathic pulmonary fibrosis (IPF) and other f-ILD, resulting in small margins of change in FVC $[2,3]$. Recently, the first trial of antifibrotic medication in patients with systemic sclerosis-associated interstitial lung disease has shown a numerically small but significant lower annualised rate of FVC decline $(41 \mathrm{~mL})$ in patients treated with nintedanib compared with placebo [3].

Data regarding a possible circadian rhythm in pulmonary function are contradictory [4-6]. Diurnal variation has never been investigated in f-ILD but could have implications for the interpretation and design of clinical trials, and for monitoring in daily practice. Taking advantage of new e-health technologies $[7,8]$, we aimed to assess whether there is a diurnal variation in FVC in patients with f-ILD using home spirometry. Furthermore, we evaluated whether there was a relationship between FVC and activity as we hypothesised that exercise just before the measurement may affect FVC values.

Between December 2018 and May 2019, consecutive outpatients with f-ILD were invited to participate in this prospective, single-centre, observational study for 6 weeks. Medical ethical committee approval was obtained and all patients provided written informed consent. Our previously developed and validated home monitoring programme was used for home-based measurements [7]. Patients measured FVC twice daily with a handheld spirometer (Spirobank Smart; MIR, Rome, Italy), once in the morning and once in the afternoon. FVC measurements were excluded if only one measurement was available for that day, if the morning FVC measurement was before $06: 00 \mathrm{~h}$ or if difference from baseline FVC was $>20 \%$. In addition, steps were continuously counted using an activity tracker (Flex 2; FitBit, San Francisco, CA, USA) in blocks of $15 \mathrm{~min}$ to assess activity during the hour before FVC measurement. At baseline and after 6 weeks, patients completed the King's Brief Interstitial Lung Disease (K-BILD) questionnaire online [9]. In-hospital spirometry was performed at the start of the study and patients received standardised instructions about the home monitoring programme. Linear mixed models were used to evaluate differences between morning and afternoon measurements. Pearson correlation coefficient was used to assess correlations between study parameters ( $\mathrm{R}$ version 3.5.2; The R Foundation for Statistical Computing, Vienna, Austria). We estimated that between four and 50 patients would be needed to determine a significant difference between morning and afternoon FVC with a power of $90 \%$, assuming a total variance of $0.026 \mathrm{~L}$ and between-patient standard deviation of $0.006-0.1 \mathrm{~L}$, based on pilot data.

Of 57 invited patients, 50 patients consented to participate. The median (range) age of patients was 68 (43-79) years and $68 \%$ were male; $50 \%$ of patients had IPF, $18 \%$ chronic hypersensitivity pneumonitis and $12 \%$ nonspecific interstitial pneumonia. Other diagnoses were combined pulmonary fibrosis and emphysema $(n=4)$, fibrotic sarcoidosis $(n=3)$, unclassifiable fibrosis $(n=1)$, pleuroparenchymal fibroelastosis $(\mathrm{n}=1)$, and anti-neutrophil cytoplasmic antibody-associated vasculitis with fibrosis $(\mathrm{n}=1)$. Median FVC was $3.0(1.5-5.2) \mathrm{L}$ or $76 \%(46-119 \%)$ of predicted, median forced expiratory volume in $1 \mathrm{~s}$ $\left(\mathrm{FEV}_{1}\right) 2.4(1.4-4.0) \mathrm{L}$ or $82 \%(50-114 \%)$ of predicted and diffusing capacity of the lung for carbon

@ERSpublications

This study demonstrates a diurnal variation in FVC measured with home spirometry in patients with f-ILD, with a higher FVC in the morning than in the afternoon http://bit.ly/37SQtBK

Cite this article as: Moor CC, van den Berg CAL, Visser LS, et al. Diurnal variation in forced vital capacity in patients with fibrotic interstitial lung disease using home spirometry. ERJ Open Res 2020; 6: 00054-2020 [https://doi.org/10.1183/23120541.00054-2020]. 
monoxide $\left(D_{\text {LCO }}\right) 50 \%(16-110 \%)$ of predicted. Mean \pm SD K-BILD total score at baseline was $57.4 \pm 11$, and the breathlessness and activity domain score was $45.3 \pm 18$.

Home-based FVC measurements were available for 44 patients; one patient withdrew consent and five patients did not manage to perform consistent measurements due to cough or bad technique. In total, 2842 FVCs were analysed. Activity measurements of 37 patients were analysed; three patients did not manage to send their activity data due to technical problems. Additionally, data from patients who did not wear their activity tracker before the morning measurement were excluded.

Morning FVC was significantly higher than afternoon FVC (mean difference $36 \mathrm{~mL}, \mathrm{p}<0.001$ ). The mean difference between morning and afternoon FVC was similar for patients with IPF compared with all f-ILDs. In 33 out of 44 patients, morning FVC was numerically higher than afternoon FVC (figure 1a). Mean \pm SD difference in FVC was $1.2 \pm 1.0 \%$ of predicted. Coefficient of variation was higher for afternoon FVC compared with morning FVC (5.1\% versus $4.6 \%, \mathrm{p}=0.018)$. No diurnal variation was found for $\mathrm{FEV}_{1}$ (mean difference $7 \mathrm{~mL}, \mathrm{p}=0.35)$. Home and hospital spirometry were highly correlated $(\mathrm{r}=0.98, \mathrm{p}<0.001)$. Total variance in FVC was $0.021 \mathrm{~L}$ and between-patient standard deviation was $0.033 \mathrm{~L}$.

Median number of steps per day was 6290 (interquartile range 3752-9439). Step count was lower before morning FVC than afternoon FVC (figure 1b). Mean difference was 49 steps during 15 min before FVC measurement $(p=0.005)$ and 219 steps during the hour before FVC measurement $(p<0.001)$. Patients were relatively inactive during the $15 \mathrm{~min}$ before spirometry; $87 \%$ of patients walked $<250$ steps. Daily step count correlated with FVC $(\mathrm{r}=0.32, \mathrm{p}=0.028), D_{\mathrm{LCO}}(\mathrm{r}=0.46, \mathrm{p}=0.001)$, K-BILD total score $(\mathrm{r}=0.5$, $\mathrm{p}<0.001)$, and K-BILD breathlessness and activities domain $(\mathrm{r}=0.6, \mathrm{p}<0.001)$.

In this study, we observed a diurnal variation in FVC measured with home spirometry in patients with f-ILD, with a higher FVC in the morning than in the afternoon. In contrast, patients had a lower step

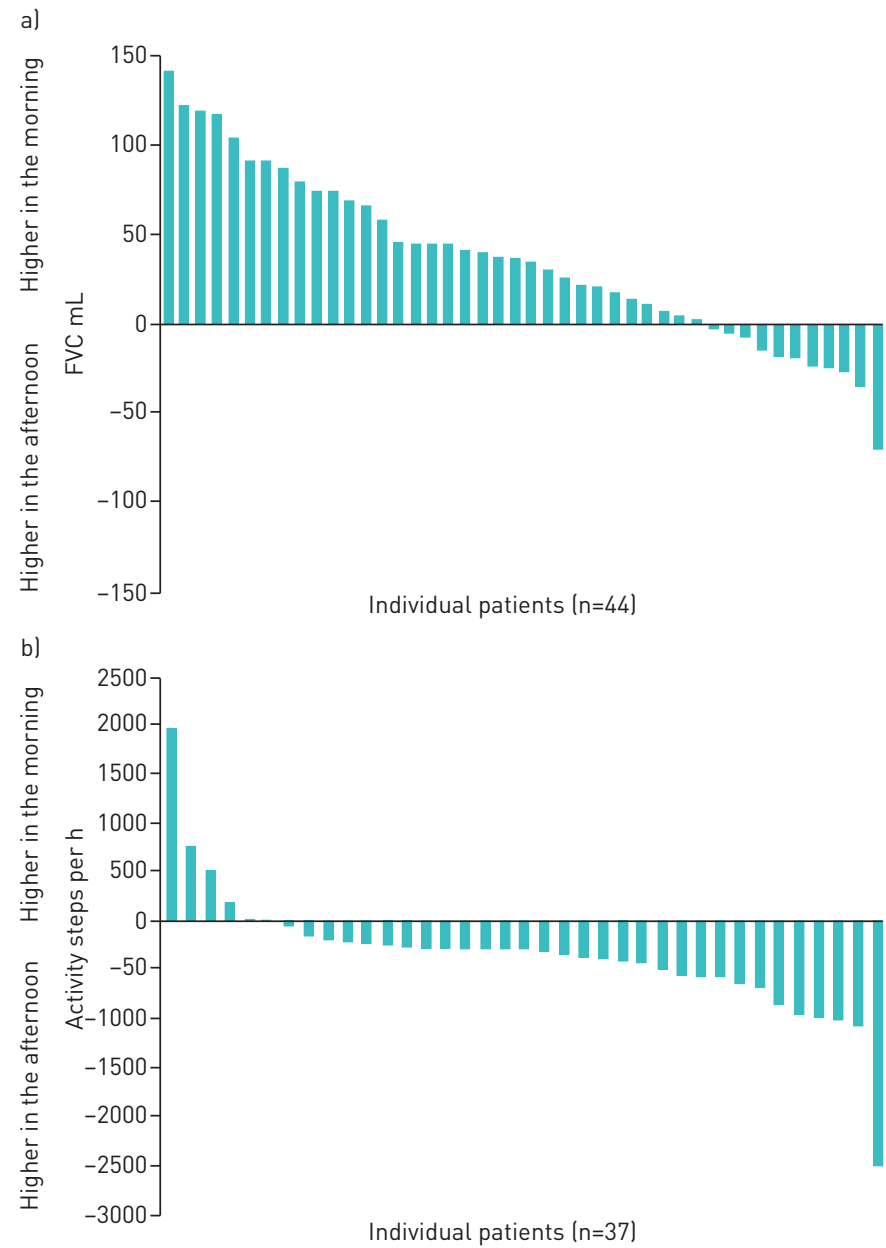

FIGURE 1 a) Differences in forced vital capacity (FVC) between morning and afternoon for individual patients. b) Differences in steps per hour before morning and afternoon FVC measurement. 
count before the measurement in the morning compared to the afternoon. However, most patients were relatively inactive before both measurements, and hence activity just before measurement cannot fully explain the diurnal variation in FVC. Most patients reported that they were more tired in the afternoon and attributed differences in FVC to fatigue.

Previous studies, mainly in asthmatics or healthy subjects, suggested that diurnal variation in lung function could be due to varying airway resistance. Proposed mechanisms are a variation in plasma cortisol level, catecholamine levels, parasympathetic tone, mucociliary clearance, and activity [4-6]. We did not observe a diurnal variation in $\mathrm{FEV}_{1}$ in our study, making variation in airway resistance less likely. Thus, the exact mechanism causing diurnal variation in FVC in patients with f-ILD remains to be elucidated.

Interestingly, steps per day had a stronger correlation with quality of life than with lung function, especially with the K-BILD breathlessness and activity domain. This finding suggests that activity better reflects how a patient feels and functions than pulmonary function alone. Home-based activity tracking could be a useful tool for future research, as our study showed that wearing an activity tracker for a relatively long period of time is feasible in patients with f-ILD.

A limitation of this study is that it was a single-centre study; hence, these findings need validation in a larger multicentre cohort. Furthermore, some patients had technical issues leading to missing data. However, in view of the large number of recordings, we believe that the impact on study outcome is limited. Compared with a recently published study using home spirometry that reported multiple technical problems, our study had very few technical issues [10]. In most previous trials with home spirometry in IPF, patients were blinded for their results. In the current study, we used an online application with direct feedback to patients and researchers, low-threshold communication with the study team, and thorough instruction of patients at baseline and during the study. Hence, home-based FVC had an acceptable variability and showed reliable results compared with hospital-based FVC. These results are encouraging for future home spirometry studies in f-ILD.

Considering the small margins in FVC change in current trials in IPF and other f-ILD [3], timing of spirometry should be standardised for research purposes. For daily care, we believe that differences between morning and afternoon FVC are too small to have an impact on serial changes and on treatment decisions.

Catharina C. Moor ${ }^{1}$, Carlijn A.L. van den Berg ${ }^{1}$, Lidewij S. Visser ${ }^{1}$, Joachim G.J.V. Aerts ${ }^{1}$, Vincent Cottin $\circledast^{2,3}$ and Marlies S. Wijsenbeek ${ }^{1}$

${ }^{1}$ Dept of Respiratory Medicine, Erasmus Medical Center, University Hospital Rotterdam, Rotterdam, The Netherlands. ${ }^{2}$ National Reference Coordinating Center for Rare Pulmonary Diseases, Louis Pradel Hospital, Lyon, France. ${ }^{3}$ Hospices Civils de Lyon, Université Claude Bernard Lyon 1, Lyon, France.

Correspondence: Marlies S. Wijsenbeek, Dept of Respiratory Medicine, Erasmus Medical Center, University Hospital Rotterdam, 's Gravendijkwal 230, Rotterdam 3015 CA, The Netherlands. E-mail: m.wijsenbeek-lourens@erasmusmc.nl

Received: 3 Feb 2020 | Accepted: 18 Feb 2020

Author contributions: All authors were involved in the design of the study. C.C. Moor, C.A.L. van den Berg, L.S. Visser and M.S. Wijsenbeek were involved in data collection. C.C. Moor, C.A.L. van den Berg and M.S. Wijsenbeek analysed the data and drafted the manuscript. L.S. Visser, J.G.J.V. Aerts and V. Cottin revised it critically for important intellectual content. All authors read and approved the final manuscript.

Conflict of interest: C.C. Moor reports grants and support from Boehringer Ingelheim outside the submitted work C.A.L. van den Berg has nothing to disclose. L.S. Visser has nothing to disclose. J.G.J.V. Aerts has nothing to disclose V. Cottin reports personal fees and nonfinancial support for advisory boards, lectures and travel to medical meetings from Actelion; grants, personal fees and nonfinancial support for consultancy, lectures and travel to medical meetings from Boehringer Ingelheim; personal fees for advisory boards and membership of a data safety monitoring boards (DSMB) from Bayer/MSD; personal fees for advisory boards and lectures from Novartis; personal fees and nonfinancial support for consultancy, lectures and travel to medical meetings from Roche; personal fees for lectures from Sanofi, personal fees for chairing as DSMB and membership of a steering committee from Promedior; personal fees for membership of a DSMB from Celgene; personal fees for advisory boards and cochairing a DSMB from Galapagos; and personal fees for membership of a DSMB from Galecto, all outside the submitted work. M.S. Wijsenbeek reports grants and other support from Hoffman-la Roche and Boehringer Ingelheim, and other support from Galapagos, all paid to her institution and all outside the submitted work.

Support statement: This study was funded by an unrestricted grant from Boehringer-Ingelheim. Funding information for this article has been deposited with the Crossref Funder Registry. 
References

1 Raghu G, Collard HR, Egan JJ, et al. An official ATS/ERS/JRS/ALAT statement: idiopathic pulmonary fibrosis: evidence-based guidelines for diagnosis and management. Am J Respir Crit Care Med 2011; 183: 788-824.

2 Maher TM, Kreuter M, Lederer DJ, et al. Rationale, design and objectives of two phase III, randomised, placebo-controlled studies of GLPG1690, a novel autotaxin inhibitor, in idiopathic pulmonary fibrosis (ISABELA 1 and 2). BMJ Open Respir Res 2019; 6: e000422.

3 Distler O, Highland KB, Gahlemann M, et al. Nintedanib for systemic sclerosis-associated interstitial lung disease. N Engl J Med 2019; 380: 2518-2528.

$4 \quad$ Hetzel MR. The pulmonary clock. Thorax 1981; 36: 481-486.

5 Medarov BI, Pavlov VA, Rossoff L. Diurnal variations in human pulmonary function. Int J Clin Exp Med 2008; 1: 267-273.

6 Borsboom GJ, van Pelt W, van Houwelingen HC, et al. Diurnal variation in lung function in subgroups from two Dutch populations: consequences for longitudinal analysis. Am J Respir Crit Care Med 1999; 159: 1163-1171.

7 Moor CC, Wapenaar M, Miedema JR, et al. A home monitoring program including real-time wireless home spirometry in idiopathic pulmonary fibrosis: a pilot study on experiences and barriers. Respir Res 2018; 19: 105.

8 Russell AM, Adamali H, Molyneaux PL, et al. Daily home spirometry: an effective tool for detecting progression in idiopathic pulmonary fibrosis. Am J Respir Crit Care Med 2016; 194: 989-997.

9 Patel AS, Siegert RJ, Brignall K, et al. The development and validation of the King's Brief Interstitial Lung Disease (K-BILD) health status questionnaire. Thorax 2012; 67: 804-810.

10 Maher TM, Corte TJ, Fischer A, et al. Pirfenidone in patients with unclassifiable progressive fibrosing interstitial lung disease: a double-blind, randomised, placebo-controlled, phase 2 trial. Lancet Respir Med 2020; 8: 147-157. 